East Asian Mathematical Journal

Vol. 29 (2013), No. 5, pp. 529-536

http://dx.doi.org/10.7858/eamj.2013.037

\title{
NEW FAMILY OF BINARY SEQUENCES WITH FOUR-VALUED CROSS-CORRELATION
}

\author{
Han-Doo Kim, Sung-Jin Cho*, Min-Jeong Kwon, and Un-Sook Choi
}

\begin{abstract}
In this paper, we find the values and the number of occurrences of each value of the cross-correlation function $C_{d}(\tau)$ when $d=$ $\frac{2^{k-1}}{2^{s}-1}\left(2^{k(i+1)}-2^{k i}+2^{s+1}-2^{k}-1\right)$, where $n=2 k, s$ is an integer such that $2 s$ divides $k$, and $i$ is odd.
\end{abstract}

\section{Introduction}

The design of binary sequences with good correlation properties are important for many research areas in communication systems. Finding the crosscorrelation between two maximal length sequences of the same period has been a meaningful research problem. For the theory of finite fields, maximal length sequences and sequences in general, we refer to [5]. The finite field with $q$ elements will be denoted by $G F(q)$. It is well known that $q=2^{k}$ for some integer $k \geq 1$. The multiplicative group of $G F(q)$ will be denoted by $G F(q)^{*}$. The group $G F(q)^{*}$ is cyclic and a primitive element of $G F(q)^{*}$ is a generator of $G F(q)^{*}$. An irreducible polynomial $f(x) \in G F(2)[x]$ of degree $n$ is primitive over $G F(2)$ if it is the minimum polynomial of some primitive element of $G F\left(2^{n}\right)$. Recall that the trace function $\operatorname{Tr}_{k}^{n}$ from the field $G F\left(2^{n}\right)$ onto the subfield $G F\left(2^{k}\right)$ is defined by $\operatorname{Tr}_{k}^{n}(x)=x+x^{2^{k}}+x^{2^{2 k}}+\cdots+x^{2^{(t-1) k}}$ where $t=n / k$. For the properties of the trace function, see [7]. Consider two binary $m$-sequences $u(t)$ and $v(t),(t=0,1, \cdots$,$) of period 2^{n}-1$. Let $\alpha$ be a primitive element of the finite field $G F\left(2^{n}\right)$. We may assume that $u(t)=\operatorname{Tr}_{1}^{n}\left(\alpha^{t}\right)$ and $v(t)=u(d t)\left(1 \leq d \leq 2^{n}-2\right)$ where $\operatorname{Tr}_{1}^{n}(x): G F\left(2^{n}\right) \rightarrow G F(2)$ is the well-known trace function and $d$ is a decimation, that is, an integer satisfying $\operatorname{gcd}\left(d, 2^{n}-1\right)=1$. The cross-correlation function $C_{d}(\tau)$ between the binary sequences $u(t)$ and $v(t)=u(d t)\left(t=0,1, \cdots, 2^{n}-2\right)$ is defined for $\tau=0,1, \cdots, 2^{n}-2$ and given by

Received December 14, 2012; Revised October 16, 2013; Accepted November 20, 2013. 2000 Mathematics Subject Classification. 97N70, 11G25, 94A55, 68Q87.

Key words and phrases. Cross-correlation, maximal length sequence, finite field, decimation, Niho's theorem.

This work was supported by the 2009 Inje University research grant.

*Corresponding author. 


$$
\begin{aligned}
C_{d}(\tau) & =\sum_{t=0}^{2^{n}-2}(-1)^{u(t+\tau)+v(t)} \\
& =\sum_{t=0}^{2^{n}-2}(-1)^{T r_{1}^{n}\left(\alpha^{t+\tau}+\alpha^{d t}\right)} .
\end{aligned}
$$

One of important problems in the theory of sequences is to determine the values and the number of occurrences of each value taken on by the crosscorrelation $C_{d}(\tau)$. Several decimations leading to three and four-valued correlation function are known.

If $d$ is not a power of two, then the cross-correlation function takes on at least three values [11].

The well known four-valued cases are:

(4a) $d=2^{n / 2+1}-1$, with $n \equiv 0(\bmod 4)$,

(4b) $d=\left(2^{n / 2}+1\right)\left(2^{n / 4}-1\right)+2$, with $n \equiv 0(\bmod 4)$,

(4c) $d=\sum_{i=0}^{n / 2} 2^{i m}$, with $n \equiv 0 \quad(\bmod 4), 0<m<n, \operatorname{gcd}(m, n)=1$,

(4d) $d=\frac{2^{k-1}}{2^{s}-1}\left(2^{2 k}+2^{s+1}-2^{k+1}-1\right), n=2 k, 2 s \mid k$,

(4e) $d=\left(\frac{p^{2 k}+1}{2}\right)^{2}$, with $n=4 k$ and $p$ an odd prime.

(4a) and (4b) were proved by Niho [8], (4c) was proved by Dobbertin [1] and (4d) was proved by Helleseth and Rosendahl [3], and Rosendahl [9]. (4e) was proved by Seo et al. [10] and Luo [6]. In 2007, Helleseth et al. [2] proposed a $d$ which has at most four-valued cross-correlation functions.

In this paper, we provide a family of decimations which lead to a four-valued cross-correlation. The decimations $d$ are as follows: $d=\frac{2^{k-1}}{2^{s}-1}\left(2^{k(i+1)}-2^{k i}+\right.$ $\left.2^{s+1}-2^{k}-1\right)$ where $n=2 k$ and $s$ is an integer such that $2 s$ divides $k$, and $i$ is odd. When $i=1$ this is equal to $(4 d)$.

\section{Preliminaries}

In this section we introduce some basic results and methods in order to completely determine the cross-correlations of the proposed four-valued sequence family. Let $n=2 k$, where $k$ is an even integer and $q=2^{k}$. For $x \in G F\left(q^{2}\right)$ we define $\bar{x}=x^{q}$. Then

(a) $\overline{x+y}=\bar{x}+\bar{y}$ and $\overline{x y}=\bar{x} \bar{y}$ for all $x, y \in G F\left(q^{2}\right)$ and

(b) $x+\bar{x} \in G F(q)$ and $x \bar{x} \in G F(q)$ for all $x \in G F\left(q^{2}\right)$.

Define the unit circle of $G F\left(q^{2}\right)$ by $S=\left\{x \in G F\left(q^{2}\right): x \bar{x}=1\right\}$. Then $S$ is the group of $(q+1)$-th roots of unity in $G F\left(q^{2}\right)$ and is a subgroup of $G F\left(q^{2}\right)^{*}$.

The following theorem is useful in finding the complete distribution of the values of $C_{d}(\tau)$. 
Theorem 2.1 $[8,11]$ For some integer $d\left(1 \leq d \leq 2^{n}-2\right)$, we have

(a) $C_{d}(\tau)$ is a real number,

(b) $\sum_{\tau=0}^{2^{n}-2}\left(C_{d}(\tau)+1\right)=2^{n}$,

(c) $\sum_{\tau=0}^{2^{n}-2}\left(C_{d}(\tau)+1\right)^{2}=2^{2 n}$

(d) $\sum_{\tau=0}^{2^{n}}-2\left(C_{d}(\tau)+1\right)^{3}=2^{2 n} b$,

where $b$ is the number of $x \in G F\left(q^{2}\right)$ such that

$$
(x+1)^{d}=x^{d}+1 .
$$

Niho [8] and Rosendahl [9] proved the following two theorems respectively.

Theorem 2.2 Let $n=2 k$ and $y \in G F\left(2^{n}\right)^{*}$. The equation

$$
x^{2^{s}+1}+y x^{2^{s}}+\bar{y} x+1=0
$$

has either $0,1,2$ or $2^{g c d(s, k)}+1$ solutions $x$ in $S$.

Theorem 2.3 Let $n=2 k$ and assume that $d \equiv 1\left(\bmod 2^{k}-1\right)$. And let $C_{d}(\tau)+1=\Delta_{d}(\tau)$. Then $\Delta_{d}(\tau)=2^{k}(N(y)-1)$ where $N(y)$ is the number of $x \in\left\{x \in G F\left(2^{n}\right): x^{2^{k}+1}=1\right\}$ such that

$$
x^{d}+y x+\bar{y} x^{-1}+x^{-d}=0
$$

and $y \in G F\left(2^{n}\right)^{*}$.

Lemma 2.4 Let $k$ be even. And let $s$ be an integer such that $2 s$ divides $k$. Then $\operatorname{gcd}\left(2^{k}+1,2^{s}-1\right)=1$.

Proof. Since $s / \operatorname{gcd}(k, s)=1$ is odd, $\operatorname{gcd}\left(2^{k}+1,2^{s}-1\right)=1$.

Lemma 2.5 Let $k$ be even. And let $s$ be an integer such that $2 s$ divides $k$. Then $\operatorname{gcd}\left(2^{k}+1,2^{s}+1\right)=1$.

Proof. Since $2 s \mid k$, let $k=2 a s$ for some integer $a$. From $2^{k}+1=2^{k-s}\left(2^{s}+\right.$ $1)-2^{k-s}+1, \operatorname{gcd}\left(2^{k}+1,2^{s}+1\right)=\operatorname{gcd}\left(2^{k-s}-1,2^{s}+1\right)$. Since $\frac{k-s}{\operatorname{gcd}(k-s, s)}=$ $\frac{k-s}{\operatorname{gcd}(k, s)}=\frac{k-s}{s}=\frac{2 a s-s}{s}=2 a-1$ is odd, $\operatorname{gcd}\left(2^{k-s}-1,2^{s}+1\right)=1$. Hence $\operatorname{gcd}\left(2^{k}+1,2^{s}+1\right)=1$.

\section{Results for four-valued cross-correlation functions}

In this section, we show a family of four-valued cross-correlation functions.

Lemma 3.1 Let $n=2 k$ and $s$ be an integer such that $2 s$ divides $k$. And let $d=\frac{2^{k-1}}{2^{s}-1}\left(2^{k(i+1)}-2^{k i}+2^{s+1}-2^{k}-1\right)$, where $i$ is odd. Then

(a) $d \equiv 1\left(\bmod 2^{k}-1\right)$,

(b) $d \equiv \frac{2^{k i}-2^{s}}{2^{s}-1}\left(\bmod 2^{k}+1\right)$,

(c) $\operatorname{gcd}\left(d, 2^{n}-1\right)=1$.

Proof. (a) Clearly $d \equiv 1\left(\bmod 2^{k}-1\right)$. 
(b) $d=2^{k-1}\left\{\frac{2^{k}-2^{s}}{2^{s}-1}\left(2^{k}-1\right)+2^{k}+1\right\} \equiv \frac{2^{k}-2^{s}}{2^{s}-1}\left(\bmod 2^{k}+1\right)$.

(c) By (a) and (b), we have $\operatorname{gcd}\left(d, 2^{n}-1\right)=\operatorname{gcd}\left(d, 2^{k}+1\right)=\operatorname{gcd}\left(\frac{2^{k i}-2^{s}}{2^{s}-1}, 2^{k}+\right.$ 1). Since $\operatorname{gcd}\left(2^{s}-1,2^{k}+1\right)=1$ by Lemma $2.4, \operatorname{gcd}\left(\frac{2^{k i}-2^{s}}{2^{s}-1}, 2^{k}+1\right)=\operatorname{gcd}\left(2^{k i}-\right.$ $\left.2^{s}, 2^{k}+1\right)$. Since $2^{k i}-2^{s} \equiv-1-2^{s}\left(\bmod 2^{k}+1\right)$, by Lemma $2.5 \operatorname{gcd}\left(2^{k i}-\right.$ $\left.2^{s}, 2^{k}+1\right)=\operatorname{gcd}\left(2^{k}+1,2^{s}+1\right)=1$. Hence $\operatorname{gcd}\left(d, 2^{n}-1\right)=1$.

Lemma 3.2 Let $q=2^{k}$ and $d \equiv 1(\bmod q-1)$. Then $x \in G F\left(q^{2}\right) \backslash\{0,1\}$ is a solution to

$$
(x+1)^{d}=x^{d}+1
$$

if and only if $x^{d-1}=(x+1)^{d-1}=1$ or $x^{d-q}=(x+1)^{d-q}=1$.

Proof. Assume that $x \in G F\left(q^{2}\right) \backslash\{0,1\}$ is a solution to Eq.(1). Since $(x+1)^{d}=x^{d}+1,(\bar{x}+1)^{d}=\left(x^{q}+1\right)^{d}=(x+1)^{q d}=\left\{(x+1)^{d}\right\}^{q}=\left(x^{d}+1\right)^{q}=$ $\bar{x}^{d}+1$. Thus $(x \bar{x}+x+\bar{x}+1)^{d}=(x \bar{x})^{d}+x^{d}+\bar{x}^{d}+1$. Since $x \bar{x} \in G F(q)$ and $x+\bar{x} \in G F(q), x \bar{x}+x+\bar{x}+1 \in G F(q)$ and thus $(x \bar{x}+x+\bar{x}+1)^{d}=x \bar{x}+x+\bar{x}+1$. Therefore we have $x \bar{x}+x+\bar{x}+1=x \bar{x}+x^{d}+\bar{x}^{d}+1$ i.e.,

$$
x+\bar{x}=x^{d}+\bar{x}^{d} .
$$

Multiplying $x^{d-q-1}$ to both sides of Eq.(2), we obtain $x^{d-q}+x^{d-1}=x^{2 d-q-1}+$ $x^{q d+d-q-1}$. Since $d \equiv 1(\bmod q-1)$, there exists a positive integer $s$ such that $d-1=(q-1) s$. Since $x^{q d+d-q-1}=x^{(q+1)(d-1)}=x^{(q+1)(q-1) s}=1$, we have $x^{2 d-q-1}-x^{d-q}-x^{d-1}+1=\left(x^{d-1}-1\right)\left(x^{d-q}-1\right)=0$. Thus we have $x^{d}=x$ or $x^{d}=x^{q}$.

(i) $x^{d}=x$ : Since $(x+1)^{d}=x^{d}+1=x+1$ and $x \in G F\left(q^{2}\right) \backslash\{0,1\}$, $(x+1)^{d-1}=1$.

(ii) $x^{d}=x^{q}$ : Since $(x+1)^{d}=\bar{x}+1=(x+1)^{q}$ and $x \in G F\left(q^{2}\right) \backslash\{0,1\}$, $(x+1)^{d-q}=1$.

Conversely, let $x^{d-1}=(x+1)^{d-1}=1$. Then $(x+1)^{d}=x+1$ and $x^{d}=x$, and thus $(x+1)^{d}=x+1=x^{d}+1$. Therefore $x$ is a solution to Eq.(1). And let $x^{d-q}=(x+1)^{d-q}=1$. Then $(x+1)^{d}=(x+1)^{q}=x^{q}+1=x^{d}+1$. Therefore $x$ is a solution to Eq.(1).

Lemma 3.3 Let $q=2^{k}$ and $d \equiv 1(\bmod q-1)$. And let $x \in G F\left(q^{2}\right)^{*}$ be a solution to $(x+1)^{d}=x^{d}+1$. Then $\left(\frac{x+1}{\bar{x}+1}\right)^{d-1}=1$ or $\left(\frac{x+1}{\bar{x}+1}\right)^{d+1}=1$.

Proof. Since every element of $G F(q)$ is a solution to $(x+1)^{d}=x^{d}+1$, we may assume that $x \neq 1$. By Lemma $3.2, x^{d}=x$ or $x^{d}=\bar{x}$. Since $(x+1)^{d}=x^{d}+1$, $(\bar{x}+1)^{d}=\bar{x}^{d}+1$. From $(x+1)^{d}(\bar{x}+1)^{d}=\left(x^{d}+1\right)\left(\bar{x}^{d}+1\right)$, we obtain $(x \bar{x}+x+\bar{x}+1)^{d}=(x \bar{x})^{d}+x^{d}+\bar{x}^{d}+1$. Since $x \bar{x} \in G F(q)$ and $x+\bar{x} \in$ $G F(q), x \bar{x}+x+\bar{x}+1 \in G F(q)$ and thus $(x \bar{x}+x+\bar{x}+1)^{d}=x \bar{x}+x+\bar{x}+1$. Therefore we have

$$
x+\bar{x}=x^{d}+\bar{x}^{d} .
$$


(i) $x^{d}=x$ : Since $\bar{x}^{d}=\bar{x}$, we have $\left(\frac{x+1}{\bar{x}+1}\right)^{d}=\frac{x^{d}+1}{\bar{x}^{d}+1}=\frac{x+1}{\bar{x}+1}$ and thus $\left(\frac{x+1}{\bar{x}+1}\right)^{d-1}=1$.

(ii) $x^{d}=\bar{x}$ : Since $\bar{x}^{d}=x$, we have $\left(\frac{x+1}{\bar{x}+1}\right)^{d}=\frac{x^{d}+1}{\bar{x}^{d}+1}=\frac{\bar{x}+1}{x+1}$ and thus $\left(\frac{x+1}{\bar{x}+1}\right)^{d+1}=1$.

Theorem 3.4 Assume that $d \equiv 1\left(\bmod 2^{k}-1\right)$. If $\operatorname{gcd}\left(d-1,2^{k}+1\right)=$ $\operatorname{gcd}\left(d+1,2^{k}+1\right)=1$, then the equation

$$
(x+1)^{d}=x^{d}+1
$$

has exactly $2^{k}$ solutions in $G F\left(2^{n}\right)$.

Proof. Since $d \equiv 1\left(\bmod 2^{k}-1\right)$, every $x \in G F\left(2^{k}\right)$ is a solution to Eq.(4). So we may assume that $x(\neq 0,1)$ is a solution to Eq.(4). Since $x$ is a solution to Eq.(4), $x^{d}=x$ or $x^{d}=\bar{x}$ by Lemma 3.2. Let $x^{d}=x$. By Lemma 3.3, $\left(\frac{x+1}{\bar{x}+1}\right)^{d-1}=1$. And let $x^{d}=\bar{x}$. By Lemma $3.3\left(\frac{x+1}{\bar{x}+1}\right)^{d+1}=1$. Since $\operatorname{gcd}(d-$ $\left.1,2^{k}+1\right)=\operatorname{gcd}\left(d+1,2^{k}+1\right)=1, \frac{x+1}{\bar{x}+1}=1$. Thus $\bar{x}=x$. Hence $x \in G F\left(2^{k}\right)$.

Lemma 3.5 Let $n=2 k$ and $s$ be an integer such that $2 s$ divides $k$. And let

$$
d=\frac{2^{k-1}}{2^{s}-1}\left(2^{k(i+1)}-2^{k i}+2^{s+1}-2^{k}-1\right),
$$

where $i$ is odd. Then

(a) $\operatorname{gcd}\left(d+1,2^{k}+1\right)=1$.

(b) $\operatorname{gcd}\left(d-1,2^{k}+1\right)=1$.

Proof. (a) Since $\operatorname{gcd}\left(2^{s}-1,2^{k}+1\right)=1$ by Lemma $2.4, \operatorname{gcd}\left(d+1,2^{k}+1\right)=$ $\operatorname{gcd}\left(\left(2^{s}-1\right)(d+1), 2^{k}+1\right)$. From $\left(2^{s}-1\right)(d+1) \equiv 2^{k-1}\left(2^{k(i+1)}-2^{k i}+2^{s+1}-2^{k}-\right.$ $1)+\left(2^{s}-1\right) \equiv-2\left(\bmod 2^{k}+1\right), \operatorname{gcd}\left(\left(2^{s}-1\right)(d+1), 2^{k}+1\right)=\operatorname{gcd}\left(2,2^{k}+1\right)=1$. Hence $\operatorname{gcd}\left(d+1,2^{k}+1\right)=1$.

(b) Since $\operatorname{gcd}\left(2^{s}-1,2^{k}+1\right)=1$ by Lemma $2.4, \operatorname{gcd}\left(d-1,2^{k}+1\right)=\operatorname{gcd}\left(\left(2^{s}-\right.\right.$ 1) $\left.(d-1), 2^{k}+1\right)$. From $\left(2^{s}-1\right)(d-1) \equiv 2^{k-1}\left(2^{k(i+1)}-2^{k i}+2^{s+1}-2^{k}-1\right)+\left(2^{s}-\right.$ $1) \equiv-2^{s+1}\left(\bmod 2^{k}+1\right), \operatorname{gcd}\left(\left(2^{s}-1\right)(d-1), 2^{k}+1\right)=\operatorname{gcd}\left(2^{s+1}, 2^{k}+1\right)=1$, Hence $\operatorname{gcd}\left(d-1,2^{k}+1\right)=1$.

By Lemma $3.1, d \equiv 1\left(\bmod 2^{k}-1\right)$. With Theorem 3.4 and Lemma 3.5, we obtain the following theorem.

Theorem 3.6 Let $n=2 k$ and $s$ be an integer such that $2 s$ divides $k$. Also let $d=\frac{2^{k-1}}{2^{s}-1}\left(2^{k(i+1)}-2^{k i}+2^{s+1}-2^{k}-1\right)$, where $i$ is odd. Then the crosscorrelation function $C_{d}(\tau)$ between two $m$-sequences takes on the following four values: 


$$
\begin{array}{cccc}
-1-2^{k} & \text { occurs } & \frac{2^{2 k+s-1}-2^{k+s-1}}{2^{s}+1} & \text { times }, \\
-1 & \text { occurs } & \frac{2^{2 k}-2^{k}-2^{s}}{2^{s}} & \text { times }, \\
-1+2^{k} & \text { occurs } & \frac{2^{2 k+s-1}-2^{2 k}-2^{k+s-1}}{2^{s}-1} & \text { times }, \\
-1+2^{k+s} & \text { occurs } & \frac{2^{2 k}-2^{k}}{2^{3 s}-2^{s}} & \text { times. }
\end{array}
$$

Proof. By Lemma $3.1 d \equiv 1\left(\bmod 2^{k}-1\right), d \equiv \frac{2^{k i}-2^{s}}{2^{s}-1}\left(\bmod 2^{k}+1\right)$ and $\operatorname{gcd}\left(d, 2^{n}-1\right)=1$. By Theorem 2.3, we obtain the following equation:

$$
\begin{aligned}
& x^{\frac{2^{k i}-2^{s}}{2^{s}-1}}+y x+\bar{y} x^{-1}+x^{-\frac{2^{k i}-2^{s}}{2^{s}-1}}=0 \\
& x^{2^{k}+1}=1 .
\end{aligned}
$$

Since $\operatorname{gcd}\left(2^{s}-1,2^{k}+1\right)=1$ by Lemma 2.4 , the number of solutions to Eq.(5) is the same as the number of solutions to the following equation replacing $x$ by $x^{2^{s}-1}$ in Eq.(5). Then we get the following:

$$
y x^{2^{s}-1}+x^{2^{k i}-2^{s}}+\bar{y} x^{1-2^{s}}+x^{-2^{k i}+2^{s}}=0 .
$$

Multiplying $x^{2^{k i}-2^{s}}$ to both sides, we obtain the equivalent equation:

$$
y x^{2^{s}-1}+x^{2\left(2^{k i}-2^{s}\right)}+\bar{y} x^{2^{k i}-2^{s+1}+1}+1=0 .
$$

Since $2^{k} \equiv-1\left(\bmod 2^{k}+1\right)$, Eq.(7) is equivalent to the following:

$$
x^{2\left(-1-2^{s}\right)}+y x^{-2}+\bar{y} x^{-2^{s+1}}+1=0 .
$$

Thus the number of solutions to Eq.(8) is the same as the number of solutions to Eq.(9):

$$
x^{-1-2^{s}}+y x^{-1}+y^{2^{k}} x^{-2^{s}}+1=0,
$$

Therefore the number of solutions to Eq.(9) is the same as the number of solutions to the following Eq.(10):

$$
x^{2^{s}+1}+y x^{2^{s}}+y^{2^{k}} x+1=0 .
$$

Thus by Theorem $2.2 C_{d}(\tau)$ is four-valued. By Theorem 2.1 and Theorem 3.4,

$$
\sum_{\tau=0}^{2^{n}-2}\left(\Delta_{d}(\tau)\right)^{3}=2^{2 n} 2^{k} .
$$

As usual, denote by $N_{j}$ the number of times Eq.(10) has exactly $j$ solutions in $S$. Then we have the following: 


$$
\begin{array}{ccc}
N_{0}+N_{1}+N_{2}+N_{2^{s}+1} & = & 2^{n}-1 . \\
-2^{k} N_{0}+0 \cdot N_{1}+2^{k} N_{2}+2^{k+s} N_{2^{s}+1} & = & 2^{n} . \\
2^{n} N_{0}+0 \cdot N_{1}+2^{n} N_{2}+2^{n+2 s} N_{2^{s}+1} & = & 2^{2 n} . \\
-2^{n+k} N_{0}+0 \cdot N_{1}+2^{n+k} N_{2}+2^{n+k+3 s} N_{2^{s}+1} & = & 2^{2 n+k} .
\end{array}
$$

Solving this system, we obtain the following: $N_{0}=\frac{2^{2 k+s-1}-2^{k+s-1}}{2^{s}+1}, N_{1}=$ $\frac{2^{2 k}-2^{k}-2^{s}}{2^{s}}, N_{2}=\frac{2^{2 k+s-1}-2^{2 k}+2^{k+s-1}}{2^{s}-1}, N_{2^{s}+1}=\frac{2^{2 k}-2^{k}}{2^{3 s}-2^{s}}$.

By Theorem $2.3 C_{d}(\tau) \in\left\{-1-2^{k},-1,-1+2^{k},-1+2^{k+s}\right\}$. This completes the proof.

\section{Conclusion}

In this paper we proposed four-valued cross-correlation functions between two maximal linear recursive sequences and found the values and the number of occurrences of each value of $C_{d}(\tau)$ when $d=\frac{2^{k-1}}{2^{s}-1}\left(2^{k(i+1)}-2^{k i}+2^{s+1}-2^{k}-1\right)$, where $n=2 k$ and $s$ is an integer such that $2 s$ divides $k$, and $i$ is odd.

\section{References}

[1] H. Dobbertin, One-to-one highly nonlinear power functions on $G F\left(2^{n}\right)$, AAECC Applicable Algebra in Engineering, Communication and Computing, Vol. 9, 1998, pp. 139-152.

[2] T. Helleseth, J. Lahtonen and P. Rosendahl, On Niho type cross-correlation functions of $m$-sequences, Finite Fields and Their Applications, Vol. 13, No. 2, 2007, pp. 305-317.

[3] T. Helleseth and P. Rosendahl, New pairs of $m$-sequences with 4-level cross-correlation, Finite Fields and Their Applications, Vol. 11, 2005, pp. 647-683.

[4] H.D. Kim and S.J. Cho, A new proof about the decimations with Niho type five-valued cross-correlation functions, J. Appl. Math. and Informatics, Vol. 30, No. 5-6, 2012, pp. 903-911.

[5] R. Lidl and H. Niederreiter, Finite fields, Cambridge University Press, 1997.

[6] J. Luo, Cross correlation of nonbinary Niho-type sequences, ISIT 2010, Austin, Texas, U.S.A., June 13-18, 2010, pp. 1297-1299.

[7] R. McEliece, Finite fields for computer scientists and engineers, Kluwer Academic Publishers, Boston, 1987.

[8] Y. Niho, Multi-valued cross-correlation functions between two maximal linear recursive sequences, Ph.D. thesis, University of Southern California, 1972.

[9] P. Rosendahl, Niho type cross-correlation functions and related equations, Ph.D. thesis, Turku center for computer science, 2004.

[10] E.Y. Seo, Y.S. Kim, J.S. No and D.J. Shin, Cross-correlation distribution of $p$-ary $m$ sequence of period $p^{4 k}-1$ and its decimated sequences by $\left(\frac{p^{2 k}+1}{2}\right)^{2}$, IEEE Trans. Inf. Theory, Vol. 54, No. 7, 2008, pp. 3140-3149.

[11] H.M. Trachtenberg, On the cross-correlation functions of maximal linear sequences, Ph.D. thesis, University of Southern California, 1970. 
HAN-DOO KIM

Institute of Basic Science and Department of Applied Mathematics, Inje University, GimHae 621-749, Korea

E-mail address: mathkhd@inje.ac.kr

SUNG-JiN CHO

Department of Applied Mathematics, Pukyong National University,

Busan 608-737, Korea

E-mail address: sjcho@pknu.ac.kr

Min-JEONG KWON

Department of Applied Mathematics, Pukyong National University, Busan 608-737, Korea

Un-SoOK ChOI

School of Free Major, Tongmyoung University, Busan 608-711, Korea 